\title{
A Comparative Analysis of SC-FDMA and OFDMA over WiMAX Broadband Networks
}

\author{
Rajesh Kumar Mewada \\ M. Tech, Research Scholar \\ Bhopal Institute of Technology, Bhopal India
}

\author{
Ashutosh Sharma, Ph.D. \\ Principal \\ Globus Engineering College, Bhopal India
}

\begin{abstract}
WiMAX, is the modern technology for providing broadband wireless access over long distance communication here we are adopted orthogonal frequency division multiple access (OFDMA) and single carrier frequency division multiple access (SC-FDMA) scheme for the uplink transmissions, SCFDMA utilizes single carrier modulation and frequency domain equalization. In this paper, we are presenting a Raised Cosine and Root raised cosine scheme to reduce the peak-toaverage power ratio (PAPR) of SCFDMA signals. The current scheme can transform the original SC-FDMA signals into Raised Cosine and Root Raised Cosine like distributed. Moreover, the scheme use IFDMA for compress the large signals and repetition while DFDMA for allocating different subcarriers. Computer simulation results show that the proposed SC-FDMA scheme can offer better PAPR compare to OFDMA.
\end{abstract}

Keywords

WIMAX, OFDM, OFDMA, SCFDMA, PAPR.

\section{INTRODUCTION}

WiMAX (Worldwide Interoperability for Microwaves Access) is a Broadband 3G/4G technology. Which is based upon IEEE 802.16 standard that has been developed by working group number 16 of IEEE 802, specializing in Broadband Wireless Access the IEEE 802.16 standard allocate good QoS which is supported by a various companies[1]. The IEEE 802.16 standard for wireless use in metropolitan area network (WMAN) in 2004, the IEEE 802.16d standard was design for fixed wireless access (FWA) application. In December 2005 the IEEE 802.16e amendment, this aimed to support mobile wireless access (MWA) with seamless network coverage. This standard is now receiving notable industrial attention. Later the standard extended its operation through different PHY specification to $2-11 \mathrm{GHz}$ frequency band enabling non line of sight (NLOS) connection [2].To analyze the performance, for each level, the received signal data was comparable to the original information. The most of the previous work related to WiMAX uplink has been mainly focused on implementation problems in the physical layer proposed raised cosine pulse shaping and root raised cosine pulse shaping methods that compare PAPR characteristics using the complementary cumulative distribution function (CCDF) for different subcarrier mapping [3]. Here the raised cosine pulse shaping method provides lower PAPR reduction as compared to root raised cosine method. PAPR study is of major importance in case battery power consumption [4]. Pulse shaping is required for a single carrier system to band limit the transmit signal. This paper show a graphical analysis of the PAPR of SCFDMA when root raised cosine (RRC) filter is considered. RRC is normally used as the transmit and receive filter in a digital
Communication system to perform matched filtering [5]. The joint response of two such filters is that of the raised cosine filter. We analytically derive the time and frequency domain WiMAX uplink. In this paper a model implementation is MATLAB on which PAPR calculation for two digital modulation schemes like QPSK, and 16QAM. The RRC filter and RC filter issued to improve PAPR performance of signal. To evaluate the performance Simulation results show that the SCFDMA has a significantly lower PAPR compared to OFDMA at different subcarrier mapping [6]. Also the result of the plot of the PAPR which provide information about the systems performance In order to solve the high PAPR problem seen in the uplink of WiMAX, experimentation is now addressing techniques such as a SCFDMA.

\section{OVERVIEW}

\subsection{Problem Statement}

In literature survey we found that WiMAX uplink has faces two major problems first the uplink passes through the higher PAPR which causes the excessive battery consumption and second is the system through put is low.

\subsection{OFDM}

OFDM is based on a multicarrier modulation technique which, in turn, is based on the concept of dividing incoming data streams of high bit rates into several data streams of lower bit rates. OFDM modulates each stream onto separate carrier frequencies, known as subcarriers. Multicarrier Modulation (MCM) techniques use guard band to in order eliminate or reduce the ISI [7]. The idea of OFDM, is slightly different from that of MCM. In OFDM, subcarriers are placed in such a manner that they are orthogonal to each other. Consequently the Inter Carrier Interference (ICI) is reduced and the available bandwidth is used more efficiently. The use of OFDM saves bandwidth as compared to the Frequency Division Multiplexing (FDM) The orthogonal overlapping nature of OFDM subcarriers not only reduces the ISI but also saves the bandwidth of system which is different from FDM where ISI is reduced by the introduction of guard bands. The addition of guard band is the wastage of power and bandwidth.

\subsection{OFDMA}

The key concept of OFDMA is that the sub carriers can overlap with each other in a way that crosstalk is avoided. This is achieved by making the sub carriers orthogonal to each other. As a result, we can send data from different users overlapping each other in parallel sub carriers, which save bandwidth we can see that with OFDMA we can save bandwidth [8]. Orthogonality not only saves the bandwidth but also removes interference between multiple sub carriers. In frequency domain the super imposed carriers are represented by SINC functions and if each sub carrier is detected at the center frequency of that explicit sub carrier, due to orthogonality, the only signal that will be detected will 
correspond to that specific sub carrier. For this to happen, the sub carriers $n$ the time domain have to be integer multiples of the base sinusoid with complete cycles per symbol time [9]. This ensures that the dot product of any different sub carrier frequencies will arrival to zero, which means the sub carriers are orthogonal.

\subsection{SC-FDMA}

SC-FDMA also deals with multiple users to share a communication resource. Its structure is alike OFDMA with an addition of Discrete Fourier Transform (DFT) block. The data symbols first pass through DFT block then are modulated on subcarriers [10]. At recipient side, the equalization is achieved by Fast Fourier Transform (FFT) estimation. As SCFDMA is acquired, from OFDMA and has same basic structure it also raises the capacity of users by using several frequencies for carrying data of a single user.

\subsection{PAPR}

The PAPR is the relation between the maximum powers of a sample in a given OFDM transmit symbol divided by the average power of that OFDM symbol. PAPR be exist when in a multicarrier system the different sub-carriers are out of phase with each other. At every extant they are different with respect to each other at different phase values. When all the points achieve the maximum value simultaneously, this will support the output envelope to abruptly shoot up which causes a 'peak' in the output envelope [11]. Owing to presence of large number of independently modulated subcarriers in an OFDM system the peak value of the system might be very high as compared to the average of the all system. This ratio of the PAPR value is termed as Peak-to- Average Power Ratio.

\section{SC-FDMA SYSTEM MODEL}

The system model for SCFDMA is shown in Figure 4. Here first we are generating data blocks and chosen a modulation technique and then to select subcarrier mapping scheme like DFDMA or IFDMA Here the pulse shaping method we used Raised Cosine (RC) and Square Root Raised Cosine (RRC) filters because they make the receiver compatible against timing synchronization errors [12].

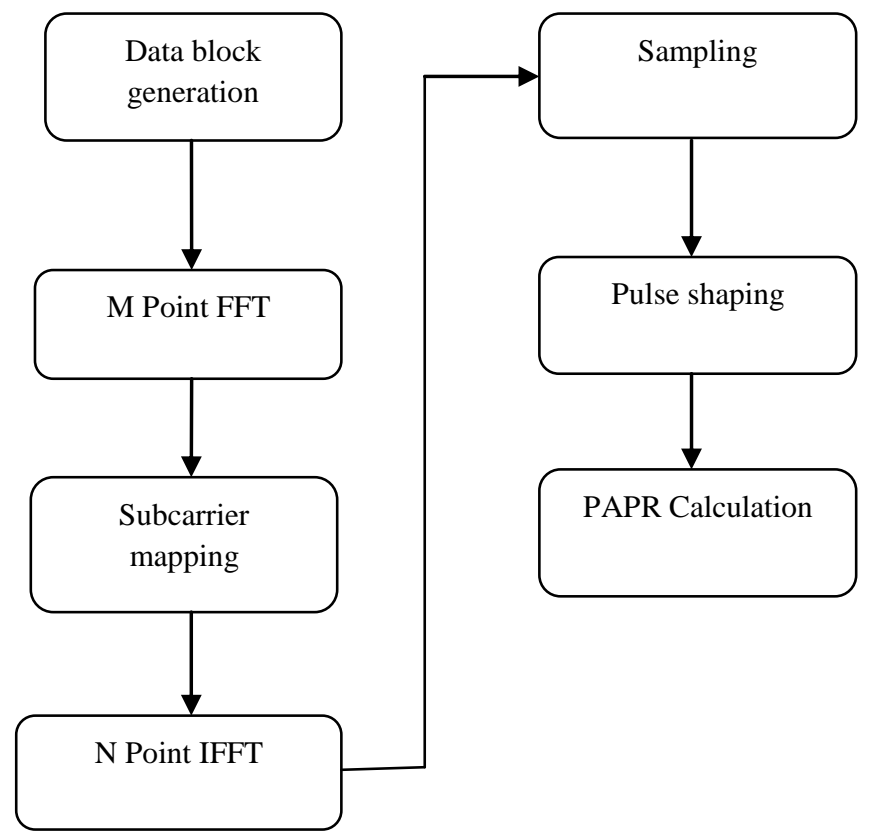

Fig: 1 SCFDMA System Model

\section{COMPUTER SIMULATIONS RESULT}

Complementary cumulative distribution function (CCDF) of PAPR, which is the probability that PAPR is higher than a certain PAPR value PAPR0, is calculated by Monte Carlo simulation. The parameters used for the calculation of PAPR are illustrated in Table 1.

Table 1 The system parameters used for simulation.

\begin{tabular}{|c|c|}
\hline System Parameter & Specification \\
\hline System bandwidth & $5 \mathrm{Mhz}, 10 \mathrm{Mhz}$ \\
\hline Number of subcarriers & 512,1024 \\
\hline Data block size & 16,32 \\
\hline Roll of factor & 0.09999999 \\
\hline Oversampling factor & 4 \\
\hline Number of iteration & $10^{4}$ \\
\hline Subcarrier mapping schemes & DFDMA,IFDMA \\
\hline Modulation data type & $16 \mathrm{QAM}, \mathrm{QPSK}$ \\
\hline Spreading factor for IFDMA & 32 \\
\hline Spreading factor for DFDMA & 31 \\
\hline
\end{tabular}

To show the overall performance of the proposed SC-FDMA scheme on reducing PAPR, we assume that randomly generated data are 16-QAM and QPSK modulated and the number of subcarriers is $\mathrm{N}=512$ and 1024 respectively.

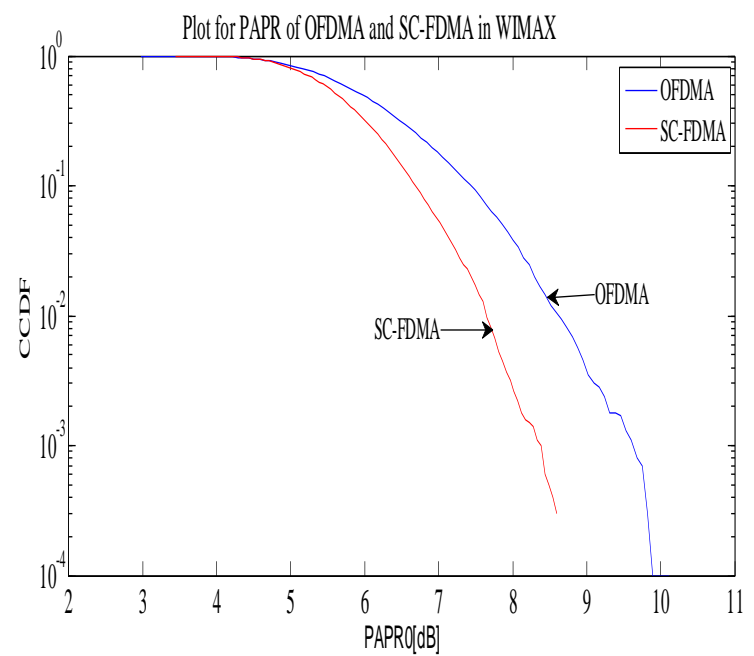

Fig.2 For block size 16, subcarrier 512 PAPR comparison of SCFDMA subcarrier mapping with OFDMA using 16QAM.

Fig.2 Describe the comparison of complementary cumulative function (CCDF) between SC-FDMA and the OFDMA scheme. It indicates that at given CCDF $=10^{-4}$, the PAPR of OFDMA signal with $\mathrm{N}=512$ is $9.87 \mathrm{~dB}$, while it is $8.87 \mathrm{~dB}$ under our proposed scheme. Our scheme has about $1 \mathrm{~dB}$ improvement over OFDMA to reduce PAPR. 


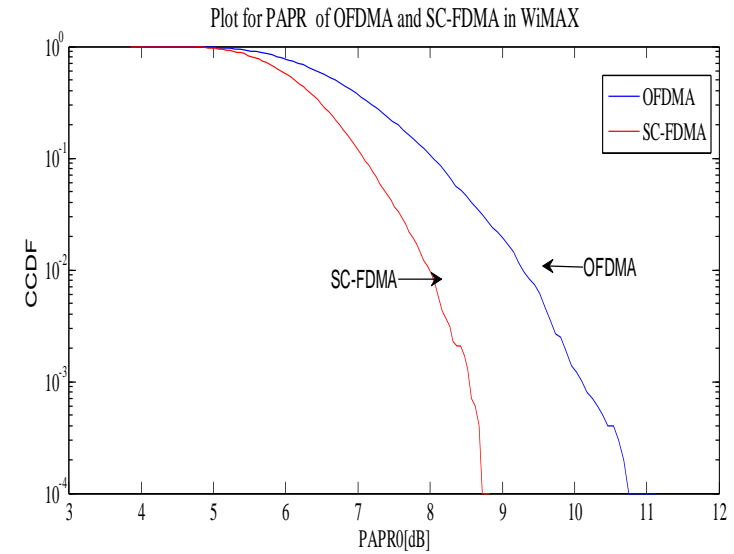

Fig.3.For block size 32, subcarrier 1024PAPR comparison of SCFDMA subcarrier mapping with OFDMA using 16QAM.

Fig, 3 explain the CCDF comparison of OFDMA and the proposed scheme SCFDMA with $\mathrm{N}=1024$. Indicate that at given $\mathrm{CCDF}=10^{-4}$, the PAPR of OFDMA signal with $\mathrm{N}=$ 1024 was $10.87 \mathrm{~dB}$, while it is $8.87 \mathrm{~dB}$ under our proposal. Our scheme has about $2 \mathrm{~dB}$ improvement in PAPR reduction.

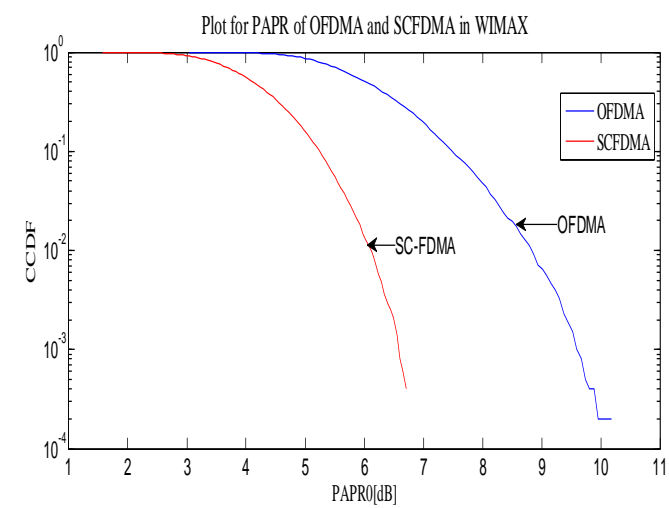

Fig.4. For block size 16, subcarrier 512 PAPR comparison of SCFDMA subcarrier mapping with OFDMA using QPSK.

Fig.4 shows the CCDF, for OFDMA and the proposed scheme SCFDMA with $\mathrm{N}=512$. Which indicates that at given CCDF $=10^{-4}$, the PAPR of OFDMA signal with $\mathrm{N}=512$ was $10 \mathrm{~dB}$, while it is $6.8 \mathrm{~dB}$ under our proposal. Our scheme has about $3.2 \mathrm{~dB}$ improvement in PAPR reduction.

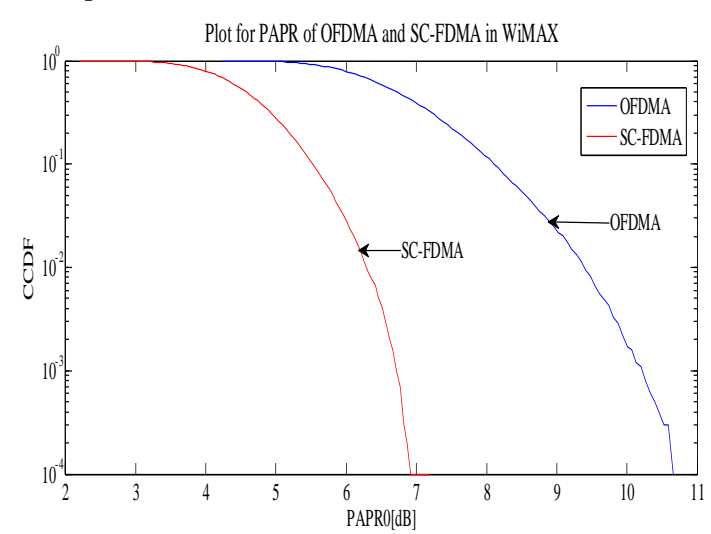

Fig.5.For block size 32, subcarrier 1024 PAPR comparison of SCFDMA subcarrier mapping with OFDMA using QPSK.
Similarly fig.5 illustrate the CCDF comparison of OFDMA and the proposed scheme SCFDMA with $\mathrm{N}=1024$. That indicates that at given $\mathrm{CCDF}=10^{-4}$, the PAPR of OFDMA signal with $\mathrm{N}=1024$ was $10.7 \mathrm{~dB}$, while it is $6.9 \mathrm{~dB}$ under our proposal. Our scheme has about $3.8 \mathrm{~dB}$ improvement in PAPR reduction.

\section{CONCLUSION}

In this research paper, the implementation of SCFDMA over WiMAX PHY layer and studied the comparison of two multiple access technique that is OFDMA and SCFDMA. We have implemented the SCFDMA and OFDMA technique to reduce the PAPR on UPLINK of PHY layer in WiMAX model using MATLAB simulation software. As the Peak to average power ratio has been reduced the throughput during the transmission is increased due to increase in throughput the time delay during the communication is reduced and consumption of battery will be less. In the graphical study we found that SCFDMA provides a lower PAPR and higher system throughput at different subcarriers compared to OFDMA.

\section{ACKNOWLEDGMENTS}

I would like to say thanks to my Director "Dr. A.K. Singh" and guide "Dr. Ashutosh Sharma", who gives their knowledge and time in order to complete this research paper. This research paper will never complete without the support faculty members of ECE departments of Bhopal Institute of Technology, Bhopal.

\section{REFERENCES}

[1] Mukesh patidar, Rupesh Dubey and Nitin kumar jain, 2012 "Performance Analysis of WimAX 802.16e Physical Layer Moodel", 978-1-4673-19898/12/\$31.00@ IEEE.

[2] IEEE 802.16-2004, "IEEE standard for local and Metropolitans Area Network. Part-16; Air Interface for Fixes Broadband Wireless Access Systems." Rev. of IEEE 802.16-2001, pp 1-857, oct 2004.

[3] M. Nadeem khan, S. Ghauri. 2008 "The WiMAX 802.16e physical layer model", IET International conference on volume, pp 117-120, 2008.

[4] M.M Rana, Md. Saiful Islam, Abbas Z. Kouzani, 2010 "Peak to Average Power Ratio Analysis for LTE Systems, "Presented Second International Conference on Communication software and Networks IEEE computer society.

[5] Enchang Sun, Ruizhe Yang, Pengbo SI, Yanhua Sun and Yanhua Zhang, 2010 "Raised Cosine like-Companding scheme for peak to average power ratio reduction of SCFDMA signals," Published in IEEE conference.

[6] Elias Yaacoub and Zaher Dawy, "A Comparison of Uplink Scheduling in OFDMA and SCFDMA," $17^{\text {th }}$ International Conference on Telecommunications in IEEE 2010.

[7] Syed Hamid ali Shah, Mudsar Iqbal, Tassadaq Hussain "Comparison between WiMAX and 3GPP LTE" Blekinge Institute of Technology August 2009 et al.

[8] Sertac sayin "PAPR reduction in OFDM communication with generalized discrete Fourier transform" njit university 2009.

[9] Vivek Kumar Jaiswal, Mr. G.S. Tripathi, "Performance Evaluation of WiMAX Based OFDMA System In 
Multipath Fading Channel," International Journal of Advanced Research in Electrical, Electronics and Instrumentation Engineering Vol. 2, Issue 6, June 2013.

[10] M. Nadeem Khan, S. Ghauri, "The WiMAX 802.16e physical layer model," IET International conference volume,2008 pp 117-120, 2008.

[11] Arun Gangwar, Manushree Bhardwaj "An Overview: Peak to Average Power Ratio in OFDM system \& its Effect" International Journal of Communication and Computer Technologies Volume 01 - No.2, Issue: 02 September 2012.
[12] Hyung G. Myung, Junsung Lim, David J. Goodman "Peak-to-average power ratio of single FDMA signals with Pulse Shaping" The 17th Annual IEEE International Symposium on Personal, Indoor and Mobile Radio Communications (PIMRC'06).

[13] Pochun Yen, Hlaing Minn, Chia-Chin Chong "PAPR Reduction for Bandwidth-Aggregated OFDM and SCFDMA Systems" 978-1-61284-254-7/11/\$26.00 (02011 IEEE. 